\title{
Every synaptic algebra has the monotone square root property
}

\author{
David J. Foulis*, Anna Jenčová and Sylvia Pulmannová ${ }^{\dagger}$
}

\begin{abstract}
A synaptic algebra is a common generalization of several ordered algebraic structures based on algebras of self-adjoint operators, including the self-adjoint part of an $\mathrm{AW}^{*}$-algebra. In this paper we prove that a synaptic algebra $A$ has the monotone square root property, i.e., if $0 \leq a, b \in A$, then $a \leq b \Rightarrow a^{1 / 2} \leq b^{1 / 2}$.
\end{abstract}

Key Words: synaptic algebra, order-unit norm, monotone square root property, commutative set, C-block, state.

AMS Classification 47B15 (81P10)

\section{Introduction}

Synaptic algebras, which are generalizations of the self-adjoint part of an $\mathrm{AW}^{*}$-algebra and of a Rickart $\mathrm{C}^{*}$-algebra, were introduced in [3] and further studied in [4, 6, 7, 8, 9, 10, 11, 18. For additional examples of synaptic algebras, see the cited references. Synaptic algebras provide natural representations for notions such as observables and states featured in the study of the mathematical foundations of quantum mechanics [12].

*Emeritus Professor, Department of Mathematics and Statistics, University of Massachusetts, Amherst, MA; Postal Address: 1 Sutton Court, Amherst, MA 01002, USA; foulis@math.umass.edu.

†Mathematical Institute, Slovak Academy of Sciences, Štefánikova 49, SK-814 73 Bratislava, Slovakia; pulmann@mat.savba.sk. The second and third authors were supported by Research and Development Support Agency under the contract No. APVV0178-11 and grant VEGA 2/0059/12. 
Each synaptic algebra $A$ is a partially ordered real linear subspace of a corresponding real linear associative algebra $R$ with unit element 1 . The algebra $R$ is called the enveloping algebra of $A$, and it is assumed that $1 \in A$ and that $A$ is an order-unit normed space with order unit 1 [1, pp. 67-69]. The positive cone in $A$ is denoted by $A^{+}=\{a \in A: 0 \leq a\}$.

We assume in what follows that $A$ is a synaptic algebra with enveloping algebra $R$ [3, Definition 1.1]. To avoid trivialities, we assume that $1 \neq 0$, which enables us to identify each $\lambda \in \mathbb{R}$ (the ordered field of real numbers) with the element $\lambda 1 \in A$. Also in what follows, the notation 'iff' abbreviates 'if and only if' and ':=' means 'equals by definition.'

Let $a, b \in A$. Then it is understood that the product $a b$ is calculated in the enveloping algebra $R$ and that it may or may not belong to $A$. But if $a$ commutes with $b$, in symbols $a C b$, then $a b=b a \in A$. In particular, $a^{2} \in A$, and $A^{+}=\left\{a^{2}: a \in A\right\}$.

If $a \in A^{+}$, there exists a unique $a^{1 / 2} \in A^{+}$- the square root of $a$ such that $\left(a^{1 / 2}\right)^{2}=a$. The absolute value of $a$ is denoted and defined by $|a|:=\left(a^{2}\right)^{1 / 2}$. Clearly, $a \in A^{+}$iff $a=|a|$.

Our purpose in this paper is to prove that $A$ has the following monotone square root (MSR) property:

$$
\text { If } a, b \in A^{+} \text {and } a \leq b \text {, then } a^{1 / 2} \leq b^{1 / 2} \text {. }
$$

We note that, by [15, Proposition 4.2.8], the self-adjoint part of a $\mathrm{C}^{*}$-algebra has the MSR property. The MSR property plays an important role, for instance, in the study of vector lattices in operator algebras [11, 19].

1.1 Remark. According to [11, Lemma 3.3], if $a, b \in A^{+}$and $a C b$, then $a \leq b \Leftrightarrow a^{1 / 2} \leq b^{1 / 2}$. Thus the MSR property holds in the special case when the elements involved commute.

\section{Preliminaries}

In this section we attend to some definitions, notation, and facts that will be needed for our proof that $A$ has the MSR property (Section 4 below).

The order-unit norm of $a \in A$ is denoted and defined by

$$
\|a\|:=\inf \{0<\lambda \in \mathbb{R}:-\lambda \leq a \leq \lambda\}
$$


(Recall that $\lambda$ is identified with $\lambda 1$.) In what follows, limits calculated in $A$ are understood to be limits with respect to the norm $\|\cdot\|$. We shall need the properties of the norm as per the following lemma.

2.1 Lemma. Let $a, b \in A^{+}$. Then: (i) $a \leq b \Rightarrow\|a\| \leq\|b\|$. (ii) $\left\|a^{1 / 2}\right\|=$ $\|a\|^{1 / 2}$. (iii) $A^{+}$is norm closed.

Proof. Part (i) follows from [13, Proposition 7.12 (c)], (ii) is a consequence of [3, Lemma 1.7 (ii)], and (iii) follows from [3, Theorem 4.7 (iii)].

If $a, b \in A$, then $a b a \in A$ and the quadratic mapping $b \mapsto a b a$ is both linear and order preserving on $A$ [3, Theorem 4.2].

An idempotent element $p=p^{2} \in A$ is called a projection and the set of all projections in $A$ is denoted by $P$. Partially ordered by the restriction of the partial order on $A$, it turns out that $P$ is an orthomodular lattice (OML) with $p \mapsto p^{\perp}:=1-p$ as the orthocomplementation [3, §5].

An element $a \in A$ is invertible iff $a$ has a (necessarily unique) inverse $a^{-1} \in A$ such that $a a^{-1}=a^{-1} a=1$.

2.2 Lemma. Let $a, b \in A$. Then:

(i) a is invertible iff there exists $0<\epsilon \in \mathbb{R}$ such that $\epsilon \leq|a|$.

(ii) If $0 \leq a$ and $a$ is invertible, then $0 \leq a^{-1}$ and $a^{1 / 2}$ is invertible.

(iii) If $0 \leq a \leq b$ and $a$ is invertible, then $b$ is invertible and $0 \leq b^{-1} \leq a^{-1}$.

Proof. (i) holds by [3, Lemma 7.2]. To prove (ii), assume that $0 \leq a$. Then $0 \leq a^{-1}$ by [3. Lemma 7.1]. Also, by (i), there exists $0<\epsilon \in \mathbb{R}$ with $\epsilon \leq a$, and since $\epsilon C a$, it follows from Remark 1.1 that $\epsilon^{1 / 2} \leq a^{1 / 2}$, so $a^{1 / 2}$ is invertible.

(iii) In our proof of part (iii), we use properties of quadratic mappings and we also use the fact that if $e \in A$ and $0 \leq e \leq 1$, then $0 \leq e^{2} \leq e[\underline{3}$, Lemma 2.5 (i)]. So assume that $0 \leq a \leq b$ and $a$ is invertible. Then $b$ and $b^{1 / 2}$ are invertible by (i) and (ii), whence $0 \leq b^{-1 / 2} a b^{-1 / 2} \leq b^{-1 / 2} b b^{-1 / 2}=1$, and therefore $0 \leq\left(b^{-1 / 2} a b^{-1 / 2}\right)^{2}=b^{-1 / 2} a b^{-1} a b^{-1 / 2} \leq b^{-1 / 2} a b^{-1 / 2}$. Multiplying the latter inequality on both sides, first by $b^{1 / 2}$, then by $a^{-1}$, we obtain $b^{-1} \leq a^{-1}$. 
Let $a \in A$ and $B \subseteq A$. We define $C(a):=\{b \in A: a C b\}, C(B):=$ $\bigcap_{b \in B} C(b)$, and $C C(B):=C(C(B))$. The subset $B$ of $A$ is said to be commutative iff $a, b \in B \Rightarrow a C b$, i.e., iff $B \subseteq C(B)$. If $B$ is commutative, then so is $C C(B)$ and $B \subseteq C C(B)$. A $C$-block in $A$ is defined to be a maximal commutative subset of $A$ [5, §5]. Evidently, $B$ is a C-block in $A$ iff $B=C(B)$ and by Zorn's lemma, any commutative subset of $A$, in particular any singleton set $\{a\}$, can be extended to a C-block.

Suppose that $B \subseteq A$ is a C-block. Then $B$ is closed under the formation of square roots and inverses, $B$ is a so-called sub-synaptic algebra of $A$, 4 , Definition 2.6], and $B$ is a commutative synaptic algebra in its own right [4, Theorem 2.7].

If $A$ is a commutative synaptic algebra, then $A$ is a commutative, associative, partially ordered, Archimedean, real linear algebra with a unity element 1 that is an order unit; it is a normed linear algebra under the orderunit norm; and it may be regarded as its own enveloping algebra. By [11, Theorem 5.11], $A$ is commutative iff $A$ is a vector lattice iff the OML $P$ is a Boolean algebra. For a commutative synaptic algebra, we have the following functional representation theorem [4, Theorem 4.1].

2.3 Theorem. Suppose that the synaptic algebra $A$ is commutative, let $X$ be the Stone space of the Boolean algebra $P$, and denote by $C(X, \mathbb{R})$ the partially ordered commutative Banach algebra, with pointwise operations and partial order and with the supremum (or uniform) norm, of all continuous real-valued functions on $X$. Then there is a subalgebra $F$ of $C(X, \mathbb{R})$ such that:

(i) The Boolean algebra $P(X, \mathbb{R}) \subseteq C(X, \mathbb{R})$ of all characteristic set functions of compact open subsets of $X$ is contained in $F$.

(ii) $F$ is a commutative synaptic algebra with unit 1 (the constant function $x \mapsto 1)$ under the operations and partial order inherited from $C(X, \mathbb{R})$, and the order-unit norm on $F$ is the supremum norm.

(iii) There exists a synaptic isomorphism ([4, Definition 2.9]) $\Psi: A \rightarrow F$ of $A$ onto $F$ such that the restriction of $\Psi$ to $P$ is the Boolean isomorphism of $P$ onto $P(X, \mathbb{R})$ corresponding to Stone's representation theorem.

Since the synaptic algebra $A$ is an order-unit space, the following definition [1, p. 72] applies. 
2.4 Definition. A state on $A$ is a linear functional $\omega: A \rightarrow \mathbb{R}$ such that (1) $\omega$ is positive, i.e., $a \in A^{+} \Rightarrow 0 \leq \omega(a)$ and $(2) \omega(1)=1$. The set of all states on $A$, called the state space of $A$, is denoted by $S(A)$.

See [1, Proposition II.1.7] and [1, Corollary II.1.5] for a proof of the next theorem.

2.5 Theorem. Let $a \in A$ and let $\rho: A \rightarrow \mathbb{R}$ be a nonzero linear functional on A. Then:

(i) $a \in A^{+}$iff $0 \leq \omega(a)$ for all $\omega \in S(A)$.

(ii) $\|a\|=\sup \{|\omega(a)|: \omega \in S(A)\}$.

(iii) $\rho$ is positive iff it is bounded with $\|\rho\|=\rho(1)$.

(iv) $\rho \in S(A)$ iff $\|\rho\|=\rho(1)=1$.

As a consequence of parts (i) and (ii) of Theorem 2.5, the states on $A$ determine both the partial order $\leq$ and the norm $\|\cdot\|$ on $A$.

\section{A sufficient condition for the MSR property}

In this section we prove that if the MSR property holds for the special case in which the elements involved are invertible, then $A$ has the MSR property (Theorem 3.2 below).

3.1 Lemma. If $a \in A^{+}$and $n=1,2,3, \ldots$, then: (i) $a+1 / n \in A^{+}$. (ii) $a+1 / n$ is invertible. (iii) $\lim _{n \rightarrow \infty}(a+1 / n)^{1 / 2}=a^{1 / 2}$.

Proof. Assume that $a \in A^{+}$and that $n$ is a positive integer. Obviously, (i) holds, and as $1 / n \leq a+1 / n$, (ii) follows from Lemma 2.2 (i). Also, by Remark 1.1. $(1 / n)^{1 / 2} \leq(a+1 / n)^{1 / 2} \leq(a+1 / n)^{1 / 2}+a^{1 / 2}$, hence $(a+1 / n)^{1 / 2}+a^{1 / 2}$ is also invertible. Thus,

$$
\begin{gathered}
(a+1 / n)^{1 / 2}-a^{1 / 2}=\left[(a+1 / n)^{1 / 2}-a^{1 / 2}\right]\left[(a+1 / n)^{1 / 2}+a^{1 / 2}\right]\left[(a+1 / n)^{1 / 2}+a^{1 / 2}\right]^{-1} \\
\quad=[a+1 / n-a]\left[(a+1 / n)^{1 / 2}+a^{1 / 2}\right]^{-1}=(1 / n)\left[(a+1 / n)^{1 / 2}+a^{1 / 2}\right]^{-1} .
\end{gathered}
$$


Furthermore, as $a \leq a+1 / n$, it follows from Remark 1.1 that, in (11), $0 \leq$ $(a+1 / n)^{1 / 2}-a^{1 / 2}$. Again by Remark 1.1, we have $(a+1)^{1 / 2} \leq(n a+1)^{1 / 2}$, so

$$
\begin{gathered}
0 \leq\left(1 / n^{1 / 2}\right)(a+1)^{1 / 2} \leq(1 / n)^{1 / 2}(n a+1)^{1 / 2}=[(n a+1) / n]^{1 / 2} \\
=(a+1 / n)^{1 / 2} \leq(a+1 / n)^{1 / 2}+a^{1 / 2}
\end{gathered}
$$

whence by Lemma 2.2 (iii),

$$
0 \leq\left[(a+1 / n)^{1 / 2}+a^{1 / 2}\right]^{-1} \leq\left[\left(1 / n^{1 / 2}\right)(a+1)^{1 / 2}\right]^{-1}=n^{1 / 2}(a+1)^{-1 / 2},
$$

and therefore

$$
0 \leq(1 / n)\left[(a+1 / n)^{1 / 2}+a^{1 / 2}\right]^{-1} \leq\left(1 / n^{1 / 2}\right)(a+1)^{-1 / 2} .
$$

Combining (11) and (2), we find that

$$
0 \leq(a+1 / n)^{1 / 2}-a^{1 / 2} \leq\left(1 / n^{1 / 2}\right)(a+1)^{-1 / 2} .
$$

By (3) and Lemma 2.1(i), we infer that

$$
\left\|(a+1 / n)^{1 / 2}-a^{1 / 2}\right\| \leq\left(1 / n^{1 / 2}\right)\left\|(a+1)^{-1 / 2}\right\|,
$$

from which (iii) follows.

3.2 Theorem. Suppose that, whenever $a, b \in A^{+}$, both $a$ and $b$ are invertible, and $a \leq b$, then $a^{1 / 2} \leq b^{1 / 2}$. Then $A$ has the MSR property.

Proof. Assume the hypothesis of the theorem, suppose that $a, b \in A^{+}$with $a \leq b$, and let $n$ be a positive integer. Then $a+1 / n, b+1 / n \in A^{+}$and both are invertible by Lemma 3.1 (i) and (ii). Clearly, $a+1 / n \leq b+1 / n$, whence $(b+1 / n)^{1 / 2}-(a+1 / n)^{1 / 2} \in A^{+}$by our hypothesis, and by Lemmas 3.1 (iii) and 2.1 (iii) we have

$$
b^{1 / 2}-a^{1 / 2}=\lim _{n \rightarrow \infty}\left((b+1 / n)^{1 / 2}-(a+1 / n)^{1 / 2}\right) \in A^{+},
$$

whereupon $a^{1 / 2} \leq b^{1 / 2}$. 


\section{Proof of the MSR property}

4.1 Lemma. Let $B$ be a C-block in $A$, suppose that $\omega \in S(A)$, let $\omega_{0}$ be the restriction of $\omega$ to the commutative synaptic algebra $B$, and let $X$ be the Stone space of the Boolean algebra $P \cap B$ of projections in B. Then:

(i) There exists a subalgebra $F$ of the commutative Banach algebra $C(X, \mathbb{R})$ such that $F$ is a commutative synaptic algebra, and there is a synaptic isomorphism $\Psi: B \rightarrow F$ of $B$ onto $F$.

(ii) $\omega_{0} \circ \Psi^{-1} \in S(F)$ and $\omega_{0} \circ \Psi^{-1}$ can be extended to a bounded positive linear functional $\hat{\omega}$ on $C(X, \mathbb{R})$ with preservation of norm.

(iii) There is a Borel measure $m_{\omega}$ on $X$ such that

$$
\omega\left(\Psi^{-1}(f)\right)=\int_{X} f(x) d m_{\omega}(x) \text { for all } f \in F .
$$

(iv) Suppose that $0 \leq a \in B$, a is invertible, $f:=\Psi(a) \in F$, and $0 \leq \lambda \in \mathbb{R}$. Then $\lambda+a$ is invertible in $B, \lambda+f$ is invertible in $F$, and

$$
\omega\left(a(\lambda+a)^{-1}\right)=\int_{X} \frac{f(x)}{\lambda+f(x)} d m_{\omega}(x) .
$$

Proof. (i) Part (i) follows from Theorem 2.3.

(ii) Clearly $\omega_{0} \in S(B)$, and it follows that $\omega_{0} \circ \Psi^{-1} \in S(F)$. The existence of an extension of $\omega_{0} \circ \Psi^{-1}$ to a bounded linear functional $\hat{\omega}$ with the same norm on $C(X, \mathbb{R})$ follows from the Hahn-Banach extension theorem $[15$, Theorem 1.6.1]. Thus $\hat{\omega}(1)=\left(\omega_{0} \circ \Psi^{-1}\right)(1)=\left\|\omega_{0} \circ \Psi^{-1}\right\|=\|\hat{\omega}\|$, and by Theorem 2.5 (iv), $\hat{\omega}$ is positive.

(iii) By the Riesz representation theorem [14, p. 247, Theorem D], there is a Borel measure $m_{\omega}$ on $X$ such that, for all $f \in C(X, \mathbb{R}), \hat{\omega}(f)=$ $\int_{X} f(x) d m_{\omega}(x)$, from which $\omega\left(\Psi^{-1}(f)\right)=\int_{X} f(x) d m_{\omega}(x)$ follows.

(iv) Assume the hypotheses of (iv). As $0 \leq a \leq \lambda+a \in B$, it follows from Lemma 2.2 that $\lambda+a$ is invertible in $B$, and since $\Psi: B \rightarrow F$ is a synaptic isomorphism, $\Psi(\lambda+a)=\lambda+f$ is invertible in $F$. The integral formula in (iv) then follows upon replacing $f$ in (iii) by $\frac{f}{\lambda+f}$.

The integral formula for the square root in the proof of the next theorem is suggested by [2, (V.5) p.116]. 
4.2 Theorem. There exists a positive $\sigma$-finite Borel measure $\mu$ on $(0, \infty) \subseteq$ $\mathbb{R}$ such that, for every invertible element $a \in A^{+}$and every state $\omega \in S(A)$,

$$
\omega\left(a^{1 / 2}\right)=\int_{0}^{\infty} \omega\left(a(\lambda+a)^{-1}\right) d \mu(\lambda) .
$$

Proof. Suppose that $0<t \in \mathbb{R}$. By the substitution $\lambda=t z^{2}$ with $0 \leq z$, we find that

$$
\int_{0}^{\infty} \frac{t}{\lambda+t} \lambda^{-1 / 2} d \lambda=2 t^{1 / 2} \int_{0}^{\infty} \frac{d z}{1+z^{2}}=2 t^{1 / 2} \frac{\pi}{2}=\pi t^{1 / 2}
$$

whence

$$
t^{1 / 2}=\frac{1}{\pi} \int_{0}^{\infty} \frac{t}{\lambda+t} \lambda^{-1 / 2} d \lambda
$$

Putting $d \mu(\lambda)=\frac{1}{\pi} \lambda^{-1 / 2} d \lambda$ in (1), we obtain a positive $\sigma$-finite Borel measure $\mu$ on $(0, \infty) \subseteq \mathbb{R}$, and we may write

$$
t^{1 / 2}=\int_{0}^{\infty} \frac{t}{\lambda+t} d \mu(\lambda) \text { for } 0<t \in \mathbb{R}
$$

Now let $a$ be an invertible element in $A^{+}$, let $\omega \in S(A)$, choose a C-block $B$ with $a \in B$ and let $X$ be the Stone space of $P \cap B$. By Lemma 4.1 (i), there is a synaptic subalgebra $F$ of $C(X, \mathbb{R})$ and there is a synaptic isomorphism $\Psi$ of $B$ onto $F$. As in Lemma 4.1 (iv), we put $f:=\Psi(a)$, so that $f^{1 / 2}=\Psi\left(a^{1 / 2}\right)$. Moreover, as $a$ is invertible, so is $a^{1 / 2}$, hence also $f^{1 / 2}$, and we have $0<f^{1 / 2}(x)$ for all $x \in X$. Thus, by Lemma 4.1 (iii) with $f$ replaced by $f^{1 / 2}$ and (2),

$$
\omega\left(a^{1 / 2}\right)=\int_{X} f^{1 / 2}(x) d m_{\omega}(x)=\int_{X}\left(\int_{0}^{\infty} \frac{f(x)}{\lambda+f(x)} d \mu(\lambda)\right) d m_{\omega}(x) .
$$

Applying Fubini's theorem [14, Theorem C, p. 148] to (3), we have

$$
\omega\left(a^{1 / 2}\right)=\int_{0}^{\infty}\left(\int_{X} \frac{f(x)}{\lambda+f(x)} d m_{\omega}(x)\right) d \mu(\lambda)
$$

and combining (4) with Lemma 4.1 (iv), we obtain the desired integral formula for $\omega\left(a^{1 / 2}\right)$.

4.3 Theorem. The synaptic algebra A has the MSR property. 
Proof. Suppose that $a, b \in A^{+}$, both $a$ and $b$ are invertible, and $a \leq b$. By Theorem [3.2, it will be sufficient to prove that $a^{1 / 2} \leq b^{1 / 2}$. Let $0 \leq \lambda \in \mathbb{R}$. Then $a \leq \lambda+a, b \leq \lambda+b$, and therefore both $\lambda+a$ and $\lambda+b$ are invertible with $\lambda+a \leq \lambda+b$. Consequently, $(\lambda+b)^{-1} \leq(\lambda+a)^{-1}$ by Lemma 2.2 (iii), whence $\lambda(\lambda+b)^{-1} \leq \lambda(\lambda+a)^{-1}$, and therefore $1-\lambda(\lambda+a)^{-1} \leq 1-\lambda(\lambda+b)^{-1}$. But

$$
\begin{gathered}
1-\lambda(\lambda+a)^{-1}=(\lambda+a)(\lambda+a)^{-1}-\lambda(\lambda+a)^{-1} \\
=(\lambda+a-\lambda)(\lambda+a)^{-1}=a(\lambda+a)^{-1},
\end{gathered}
$$

likewise $1-\lambda(\lambda+b)^{-1}=b(\lambda+b)^{-1}$, and we have $a(\lambda+a)^{-1} \leq b(\lambda+b)^{-1}$. Thus, $\omega\left(a(\lambda+a)^{-1}\right) \leq \omega\left(b(\lambda+b)^{-1}\right)$ for all $\omega \in S(A)$, and by Theorem 4.2 , we infer that $\omega\left(a^{1 / 2}\right) \leq \omega\left(b^{1 / 2}\right)$. Thus $a^{1 / 2} \leq b^{1 / 2}$ by Theorem 2.5 (i).

Concluding Remarks. In [2, Exercise V.1.10], R. Bhatia outlines a proof that if $r \in \mathbb{R}$ with $0<r<1$, then the function $f(x):=x^{r}$ for $0<x \in \mathbb{R}$ is operator monotone on Hermitian matrices. Our proof with $r=1 / 2$ for a synaptic algebra is partially based on Bhatia's argument. Pioneering work on monotone functions of matrices and operators was conducted by K. Löwner in [16] and G. Pedersen gave a short proof of Löwner's operator monotone theorem in [17]. It would be interesting and significant to determine the extent to which the results of Löwner, Bhatia, Pedersen, et al. can be extended to synaptic algebras, and we hope that our work in this paper might provide a point of departure for such a project.

\section{References}

[1] Alfsen, E.M., Compact Convex Sets and Boundary Integrals, SpringerVerlag, Heidelberg, New York, 1971.

[2] Bhatia, Rajendra, Matrix Analysis, Springer-Verlag, New York Inc., 1997.

[3] Foulis, D.J., Synaptic algebras, Math. Slovaca 60 (2010) 631-654.

[4] Foulis, D.J. and Pulmannová, Sylvia, Projections in a synaptic algebra, Order 27 (2010) 235-257.

[5] Foulis, D.J. and Pulmannová, Sylvia, Regular elements in generalized Hermitian algebras, Math. Slovaca 61, no. 2 (2011) 155-172. 
[6] Foulis, D.J. and Pulmannová, Sylvia, Type-decomposition of a synaptic algebra, Found. Phys. 43, no. 8 (2013) 948-968.

[7] Foulis, D.J. and Pulmannová, Sylvia, Symmetries in synaptic algebras, Math. Slovaca 64, no. 3 (2014) 751-776.

[8] Foulis, D.J. and Pulmannová, Sylvia, Commutativity in a synaptic algebra, to appear in Math. Slovaca.

[9] Foulis, David J., Jenčová, Anna, and Pulmannová, Sylvia, Two projections in a synaptic algebra, Linear Algebra Appl. 478 (2015) 162187.

[10] Foulis, David J., Jenčová, Anna, and Pulmannová, Sylvia, A projection and an effect in a synaptic algebra, Linear Algebra Appl., 485 (2015) 417-441.

[11] Foulis, David J., Jenčová, Anna, and Pulmannová, Sylvia, Vector lattices in synaptic algebras, in preparation.

[12] Foulis, David J., Jenčová, Anna, and Pulmannová, Sylvia, States and observables on synaptic algebras, in preparation.

[13] Goodearl, K.R., Partially Ordered Abelian Groups with Interpolation, A.M.S. Math. Surveys and Monographs, No. 20, 1980.

[14] Halmos, P.R., Measure Theory, Springer-Verlag, New York Inc., 1970.

[15] Kadison, R.V. and Ringrose, J.R.. Fundamentals of the Theory of Operator Algebras I, Academic Press Inc., New York, 1983.

[16] Löwner, Karl, Über monotone Matrixfunktionen, (German) Math. Z. 38, no. 1 (1934) 177-216.

[17] Pedersen, Gert, Some operator monotone functions, Proc. A.M.S. 16, no. 1 (1972) 309-310.

[18] Pulmannová, Sylvia, A note on ideals in synaptic algebras, Math. Slovaca 62, no. 6 (2012) 1091-1104.

[19] Topping, David M., Vector lattices of self-adjoint operators, Trans. Amer. Math. Soc. 115 (1965) 14-30. 